\title{
Scales of the Extra Dimensions and their Gravitational Wave Backgrounds
}

\author{
Craig J. Hogan \\ Astronomy and Physics Departments, University of Washington, Seattle, Washington 98195-1580
}

\begin{abstract}
Circumstances are described in which symmetry breaking during the formation of our threedimensional brane within a higher-dimensional space in the early universe excites mesoscopic classical radion or brane-displacement degrees of freedom and produces a detectable stochastic background of gravitational radiation. The spectrum of the background is related to the unification energy scale and the the sizes and numbers of large extra dimensions. It is shown that properties of the background observable by gravitational-wave observatories at frequencies $f \approx 10^{-4} \mathrm{~Hz}$ to $10^{3} \mathrm{~Hz}$ contain information about unification on energy scales from 1 to $10^{10} \mathrm{TeV}$, gravity propagating through extra-dimension sizes from $1 \mathrm{~mm}$ to $10^{-18} \mathrm{~mm}$, and the dynamical history and stabilization of from one to seven extra dimensions.
\end{abstract}

\section{RELIC BACKGROUNDS FROM BRANE FORMATION IN "THE DESERT"}

If quantum gravity lies fundamentally in ten spatial dimensions, then the seven "extra" dimensions must somehow be hidden. The traditional approach, going back to Kaluza and Klein, is to make extra dimensions very small, close the Planck scale. Recently there has been a resurgence of interest in another approach where many of the extra dimensions are of much larger size [7] 12]. The Standard Model fields are confined to a three-dimensional wall or "brane", a 3-D defect in the higher-dimensional space; only gravity can propagate in the other dimensions or "bulk".

These ideas are observationally constrained from the particle-physics side and from the gravity side. Roughly speaking, the success of the Standard Model limits the unification energy scale to energies well above $1 \mathrm{TeV}$, although some of the most interesting brane+bulk scenarios are those where the unification scale is not many orders of magnitude more than a TeV, providing a solution to to the "hierarchy problem." Laboratory measurements of gravity limit the size (or radius of curvature) of the extra dimensions to be smaller than about $0.3 \mathrm{~mm}$ [13]. Constraints of a modeldependent nature, including astrophysical ones, extend these limits, since the models generally add new particles and interactions to the Standard Model (e.g., [9,14,15]). A broad range of unification scales and brane configurations is consistent with current constraints; the unification scale and extra dimension size can be anywhere between the $\mathrm{TeV} / \mathrm{mm}$ limits and the Planck scale.

This broad range of possibilities of course just reflects the relative lack of data in the physics/cosmology "desert". Standard unification ideas often show running couplings extrapolated from actual experiments at $100 \mathrm{GeV}$ by 14 orders of magnitude to the unification scale at $10^{16} \mathrm{GeV}$, but there are few direct particle data beyond the TeV scale. A similar lacuna appears in the cosmological data; we can cite direct information from cosmic abundances about microphysical processes as early as $1 \mathrm{MeV}$, which can be affected by inhomogeneities originating as early as $100 \mathrm{GeV}$ (e.g. (4) and about earlier inflationary effects on very large scales (such the microwave background anisotropy, e.g. [5]), but we have almost no direct data about early "mesoscopic" structure on comoving scales smaller than a few centimeters at $100 \mathrm{GeV}$ (a quantity of energy about equal to the mass of the Earth) at times earlier than $10^{-9}$ second (except possibly for the mean baryon-to-photon ratio and the mean density of some forms of dark matter.) Scalar metric perturbations are very effectively erased on these small scales by neutrino diffusion long before they can have any effect on observables such as element abundances 6. Small-scale perturbations in baryon/photon ratio $\eta$ on small scales, even of large amplitude, are erased by nucleon diffusion before nucleosynthesis [2 4 . From most of cosmic history (in log space) since the Planck time, and on most scales of structure, only the tensor modes - gravitational waves - survive to the present.

New gravitational wave observatories now under development will soon achieve a critical level of sensitivity at frequencies where they can plausibly detect the relic stochastic background of gravitational waves from cosmological events far into the early, previously unobservable "mesoscopic" era. One of the most interesting possible sources of radiation, from the point of view of both physics and cosmology, is the formation of our 3-brane. This paper surveys broadly the circumstances and the values of parameters - the number and the size of large extra dimensions, and the scale of unification - for which the background might be detected with current technology.

To create a detectable background, the metric during the unification era must be far from equilibrium - it must be close (within a few orders of magnitude) to chaotic on the mesoscopic scale of the horizon at unification. Such circumstances can indeed be more generic than the usual assumption of a uniform equlibrium system. The brane+bulk configurations being contemplated are classical setups: the vacua of the fundamental theory have effective potentials that tend to drive them to forming classical three-dimensional defects. The details of the stabilization of the setup are 
not known but the cosmological formation of such defects is in general not an equilibrium achieved by a microscopic quantum process - it involves macroscopic (or at least mesoscopic) collective dynamics, in the same way that spontaneous symmetry breaking occurs in the standard Higgs mechanism. In the cosmological context, symmetry breaking during the setting up of the brane is likely to create gravitational waves via mechanisms analogous to such intense classical sources as cosmic strings or first-order phase transitions. The properties of the waves can be informative about both the physics of the brane and its early cosmological history.

\section{CHARACTERISTIC FREQUENCY AND AMPLITUDE}

The formation and stabilization of the brane are associated with two new geometrical degrees of freedom, radion modes governing the size or curvature of the extra dimensions and Nambu-Goldstone modes corresponding to spatial variations in brane displacement [1]. The characteristic scales of the resulting broadband background spectra can be estimated from general scaling considerations. The characteristic amplitude of the metric perturbations induced by brane condensation is of the the order of unity and the characteristic frequency is the horizon scale. We will use the "maximal amplitude" and "Hubble frequency" to estimate the extra-dimensional scales accessible to gravitational wave detectors.

The characteristic scale for setting up our $3+1$ world is the gravitational timescale $H^{-1}$ in standard $3+1$ General Relativity. This is cleanly determined by General Relativity and thermodynamics except for a weak dependence on the particle-physics uncertainties encapsulated in the number of effective relativistic degrees of freedom $g_{*}$ (e.g. [16]). The characteristic frequency of observed gravitational waves is thus [17 19] the Hubbble frequency redshifted to the present day, $f_{H 0}(T) \equiv H(T) a(T) / a_{0}$ :

$$
f_{H 0}=7.65 \times 10^{-5} \mathrm{~Hz}(\mathrm{~T} / \mathrm{TeV}) g_{*}^{1 / 6}\left(g_{*} / g_{* S}\right)^{1 / 3} T_{2.728}=9.37 \times 10^{-5} \mathrm{~Hz}(H \times 1 \mathrm{~mm})^{1 / 2} g_{*}^{-1 / 12}\left(g_{*} / g_{* S}\right)^{1 / 3} T_{2.728}
$$

The estimate is valid back to the threshold of the extradimensional dynamics: $H^{-1} \leq b_{0}$, (where $b_{0}$ is the size or curvature radius of the largest extra dimension), or $T \geq M_{*}$, whichever comes first.

The fiducial "maximal" energy density is set 17 19] by the mean energy density in all relativistic species (photons and three massless neutrinos), $\Omega_{\text {rel }}=8.51 \times 10^{-5} h_{70}^{-2} T_{2.728}^{4}$, where $h_{70}$ refers to the Hubble constant. The energy density of relativistic matter redshifts in the same way as gravitational waves so they have the same ratio of energy densities today as when the waves were generated. (Constraints from nucleosynthesis limit the gravitational waves to about $10 \%$ or less of the relativistic matter density, or rms strain about three times smaller than the maximal value.)

At the low frequencies observed by spacecraft interferometers (such as LISA) there are many astrophysical foregrounds, including known sources such as galactic binaries. A stochastic background can however be distinguished from other sources of noise and astrophysical wave sources by resolving the background in frequency. For the projected sensitivity of LISA, a maximal background is detectable above the instrument noise or the other likely astrophysical foregrounds [1,20.21] over a frequency range from about $10^{-1}$ to $10^{-4} \mathrm{~Hz}$. In the center of this range, $\approx 10^{-4} \mathrm{~Hz}$, backgrounds are detectable with

$$
\left(\frac{\Omega_{G W}(\Delta f=f)}{\Omega_{r e l}}\right) \approx 10^{-6}
$$

At the higher frequencies observed by ground-based observatories (e.g., LIGO, VIRGO, TAMA300, GEO600) it could well be that other stochastic astrophysical backgrounds are relatively weak, and that the observable sources are so limited in time duration and frequency that the main contribution to the rms noise comes from the instrument itself. In this case we can use the entire bandwidth to measure the background and reach a level which places meaningful constraints on backgrounds [18,22]. The most promising technique is to correlate the signals from two interferometers within a wavelength of each other. Combining two early LIGO or VIRGO systems will likely achieve a sensitivity [18]

$$
\left(\frac{\Omega_{G W}(\Delta f=f)}{\Omega_{r e l}}\right) \approx 10^{-2}
$$

and LIGO II may reach another two orders of magnitude below this [23] - close to the level where other stochastic astrophysical backgrounds (such as neutron star emission) may be expected. Sufficient sensitivity to constrain primordial stochastic backgrounds may be expected for early ground-based interferometers roughly from 50 to 500 $\mathrm{Hz}$. 


\section{EXCITATION OF CLASSICAL DISPLACEMENT AND RADION PERTURBATIONS}

There are many possible "brane worlds" with various configurations of large extra dimensions. To simplify the discussion we will assume that there are $n$ equal-size largest extra dimensions of size $b_{0}$ and all the others are much smaller, of size $M_{*}^{-1}$ where $M_{*}$ is the true fundamental unification scale. The apparent (usual) Planck mass in 3+1-D, $M_{\text {Planck }}$, is approximately given by $M_{\text {Planck }}^{2} \approx M_{*}^{2}\left(M_{*}^{n} V_{n}\right)$ where $V_{n}$ is the volume of the extra dimensions. In our situation,

$$
M_{\text {Planck }}^{2} \approx M_{*}^{2}\left(M_{*}^{n} b_{0}^{n}\right)
$$

where $b_{0}^{n}$ is the volume of $n$ extra large dimensions. Thus each choice of $n$ defines a relation between $M_{*}$ and $b_{0}$, shown in Figure 1. The various relations intersect at $M_{*}=M_{\text {Planck }}$ :

$$
b_{0} \approx\left(M_{\text {Planck }} / M_{*}\right)^{2 / n} M_{*}^{-1} .
$$

Gravity is "normal" 1 on scales larger than $b_{0}$; standard field theory holds on the brane at energies below $M_{*}$.

The cosmological formation of the brane setup is regulated on the gravitational timescale. In the $3+1-\mathrm{D}$ era, this is just given by the Hubble scale discussed above,

$$
H^{-1} / 1 m m \approx(T / 1 T e V)^{-2}
$$

which defines the usual gravitational relationship between length and energy. If we follow $3+1-\mathrm{D}$ cosmology back using this equation, we come first to either (1) the time when $T=M_{*}$ and extra-dimensional effects come into play on a small scale, possibly associated with a first-order phase transition; or $(2)$ the time when $H=b_{0}^{-1}$, and the geometrical degrees of freedom of the extra dimensions are important even on large scales. In general the excitation of the extra-dimensional modes has a different character depending on whether $b_{0}\left(M_{*}\right)$ lies above or below the Hubble relation $H\left(T=M_{*}\right)$. The former case produces displacement modes at with a scale determined by $b_{0}$; the latter case produces radion modes with a scale determined by $M_{*}$.

The Hubble relation $H(T)$ is degenerate with the $n=2$ relation between $b_{0}$ and $M_{*}$, meaning that in this case macroscopic and microscopic departures from the standard picture happen at about the same time. The transition to new microscopic unification physics and to 5-dimensional cosmological expansion would both happen at the same point on these lines, also corresponding to the condensation of the brane and the creation of the gravitational waves.

In the $n=1$ case where $b_{0}>H\left(T=M_{*}\right)^{-1}$, the condensation of the brane (at $T=M_{*}$ ) takes place while the Hubble length is still much smaller than $b_{0}$. In this case a long evolution takes place after the brane forms (and non-gravitational fields are confined to 3D as today), during which the cosmological evolution involves one classically large extra dimension and the usual relativistic cosmology does not apply. In particular there is not time for signals to propagate as far as $b_{0}$ in an expansion time. If the formation of the brane spontaneously breaks Poincaré invariance of the whole spacetime, the position of the brane when it condenses is therefore uncorrelated on scales larger than $H\left(T=M_{*}\right)^{-1}$ and less than $b_{0}$. The Nambu-Goldstone modes corresponding to brane displacement are therefore substantially excited by the Kibble mechanism 24,25] up to wavelength $b_{0}$. These large-amplitude scalar perturbations dynamically couple to the tensor modes to produce a gravitational wave background, in much the same way that gravitational waves are generated by defects confined to a $3+1-\mathrm{D}$ space $1,17,22,26,28$. The spectrum is peaked at the corresponding redshifted Hubble frequency $f_{H 0}\left(H^{-1}=b_{0}\right)$ and falls off as a power law at higher and lower frequencies. The spectrum at high frequencies depends on details of the $4+1-\mathrm{D}$ era of cosmic evolution, but may be as strong as $\Omega_{G W}(\Delta f=f)=$ constant; at lower frequencies, it is estimated [1] to fall off as $\Omega_{G W}(\delta f=f) \propto f^{7}$.

In the $3 \leq n \leq 7$ case where $b_{0}<H\left(T=M_{*}\right)^{-1}$, the universe can find itself in a macroscopic space of conventional 3-D dimensionality (on the Hubble scale) at the temperature where new extra-dimensional unification physics comes into play on a microscopic scale. Conventional cosmology can therefore be used as a framework. The stabilization of the $3 \leq n \leq 7$ extra dimensions appears as a change of physics, nongravitational and gravitational, which can be described macroscopically as a change in the energy-momentum tensor of cosmic matter, and may be (3D-) spatially inhomogeneous as a result of symmetry breaking. The excitation of these "radion modes" can be described as a change in the order parameter of a vacuum state in 3D, as is familiar in cosmological phase transitions. If this transition described by the effective radion potential is first order, free energy is released in macroscopic flows created

\footnotetext{
${ }^{1}$ Gauss' law shows that gravity obeys a $r^{-2}$ law for $r>b_{0}$, and $r^{-2-n}$ for $r<b_{0}$.
} 
by bubble nucleation and collisions, and can have a substantial gravitational-wave component [29 32], although for most plausible situations these backgrounds are several orders of magnitude weaker than $\Omega_{r e l}$. Figure 1 illustrates that the phase-transition bubbles are smaller than the horizon and the peak of the spectrum lies significantly higher than the redshifted Hubble frequency.2]

\section{RANGE OF ACCESSIBLE PARAMETERS}

Although based on a simplified picture, Figure 1 illustrates accurately the wide range of $n, b, M_{*}$ potentially accessible via this technique. The possibilities include both the radion and Nambu-Goldstone displacement modes, and include, for some $M_{*}$ and $b_{0}$, all likely values of $n$. The observatories constrain theories with values of these parameters including the limits accessible by other means, near $M_{*}=1 \mathrm{TeV}$ and $b_{0}=1 \mathrm{~mm}$, and extending beyond this range by many orders of magnitude. For example, direct laboratory gravitational experiments become extremely challenging below the $\mathrm{mm}$ scale, whereas primordial gravitational wave backgrounds at the highest detectable frequencies (about $1 \mathrm{kHz}$ ) probe new classical spacetime dimensions directly (via the displacement modes) on scales 14 orders of magnitude smaller - aided by the cosmological redshift which stretches the waves to detectable wavelengths. Even a nondetection of backgrounds will constrain the behavior of theories with these numbers and sizes of extra dimensions.

Of particular interest are the models which potentially solve the hierarchy problem, where $M_{*}$ is not many orders of magnitude more than $1 \mathrm{TeV}$. For these models, backgrounds in the LISA band can arise from all the viable numbers of extra dimensions $(n=2$ to $n=7)$, and a large range of dimension sizes $-10^{-12} \mathrm{~mm} \leq b_{0} \leq 1 \mathrm{~mm}$, depending on $n$. (It is already known that the $n=1$ case requires a unification scale of at least $10^{6} \mathrm{TeV}$ to be consistent with the EotWash limits).

The LIGO/VIRGO frequencies probe much higher energy unification scales, from $M_{*} \approx 10^{4}$ to $10^{10} \mathrm{TeV}$, and $b_{0}$ less than $10^{-10} \mathrm{~mm}$. The intermediate range of $b_{0}$ and $M_{*}$, corresponding to the frequency gap between these two sets of experiments, would be obervable with a space interferometer with a smaller baseline than LISA.

The smallest dimension accessible is determined by the highest frequency, which is set by the photon noise limit of LIGO or its successors. It is reasonable to expect high sensitivity with this technology up to about $1 \mathrm{kHz}$, which probes displacement modes down to $b_{0} \approx H^{-1} \approx 10^{-14} \mathrm{~mm}$. The largest $M_{*}$ probed (corresponding to $n=1$ ) can be as high as about $10^{10} \mathrm{TeV}$; the smallest $b_{0}$ (corresponding to $n=7$ ) can be as short as $10^{-18} \mathrm{~mm}$. There are no known strong astrophysical sources of gravitational radiation above $10^{4} \mathrm{~Hz}$, so new types of experiments at higher frequency, with sufficient sensitivity (i.e. $\Omega_{G W} \leq \Omega_{r e l}$ ), would probe even more extreme physics with little astrophysical contamination.

As an aside, it is interesting to consider the traditional case with all the extra dimensions at the classical Planck scale. This point corresponds to $M_{\text {Planck }}^{-1} \approx b_{0} \approx M_{*}^{-1}$ for any value of $n$. (The relation $b_{0} \approx M_{*}^{-1}$ shown in Figure 1 for other values of $M_{*}$ corresponds to the $n=\infty$ limit of equation 5 .) The gravitational wave background in this model has a peak frequency of about $10^{12} \mathrm{~Hz}$, or a peak wavelength of about $0.3 \mathrm{~mm}$ ! Although the maximal classical background typically has $10^{10}$ times more energy density than tensor perturbations from inflationary quantum effects, ; there is no plausible technique for detecting a gravitational wave background at such high frequencies.

In models enclosed by the boxes in Figure 1. properties of these backgrounds can in some circumstances provide measurements of the main parameters of the largest extra dimensions: $n, b_{0}$ and $M_{*}$. For example, suppose that a stochastic background is detected with various signatures (such as anisotropy statistics) leading us to suspect that it comes from high redshift. If the background is within one or two orders of magnitude of $\Omega_{r e l}$ it is likely from a

\footnotetext{
${ }^{2}$ Account is not taken of the narrower instrumental bandpasses for the less-than-maximal intensity of the background in these cases.

${ }^{3}$ Most discussions of primordial tensor modes concentrate on waves excited by quantum effects at inflation. These much weaker waves are probably unobservable at LIGO and LISA frequencies, but may already be observable on much larger scales through their effect on microwave background anisotropy. A gravitational wave background is produced by quantum fluctuations of the graviton during inflation, with rms tensor-mode amplitude (e.g. [16]) $h_{t} \approx\left(H_{\text {inflation }} / M_{\text {Planck }}\right)^{2}$. This is most easily detected on comoving scales close to the present-day Hubble length, $f \approx H_{0} \approx 2 \times 10^{-18} \mathrm{~Hz}$, where background radiation anisotropy implies an amplitude $h_{t} \leq 10^{-5}$. Of course the amplitude might be very different on the scales of the $10^{-4}$ to $1000 \mathrm{~Hz}$ waves considered here, but since $H_{\text {inflation }}$ generally decreases slowly during inflation the "tilt" from a scale-free spectrum generally makes $h_{t}$ smaller at higher frequencies. The energy density of this background is $h_{t}^{2}$ times smaller than the maximal value $\left(h_{t}=1\right)$ and is likely to be unobservable.
} 
displacement mode. This in turn indicates that $n=1$ or $n=2-$ one or two extra dimensions are much larger than the rest. Since only $n=1$ undergoes a substantial period of classical expansion with $b_{0}>H^{-1}$, the background spectra in these two situations are likely to be qualitatively different: the $n=2$ spectrum is more sharply peaked at $f_{H 0}$ and the $n=1$ spectrum includes a more intense high-frequency tail from modes excited before $H_{0}^{-1}=b_{0}$. Knowing $n$, the frequency of the spectral peak then provides an estimate of $b_{0}$ and $M_{*}$. A nondection of a displacement-mode background conveys a significant constraint on parameters and on cosmology since the conditions for generating a background from symmetry breaking in this regime are relatively generic.

In the case of the $n \geq 3$ radion modes, it is difficult to break the degeneracies using just the information available from the background measurements. For example, the peak frequency and intensity depend on the details of bubble nucleation dynamics as well as on $f_{H 0}$; these in turn depend on the details of the effective radion potential. That is, a weaker transition leads to a smaller nucleation scale, a higher frequency peak and a less intense background. (The interpretation of the background might in the case of LISA-band backgrounds be supplemented by other data, since the energy scale $M_{*}$ is not too far above energies accessible by direct experiment.) A nondetection in this regime will only constrain the corresponding parameters in particular models; lack of a background could just mean that the relevant transition is not strongly first order, but occurs gently and does not generate intense gravitational waves.

\section{ACKNOWLEDGMENTS}

I am grateful for useful discussions with E. Adelberger, B. Allen, B. Barish, P. Bender, D. Kaplan, A. Nelson, and K. Thorne. I thank the Albert Einstein Institute, Golm, the LIGO Hanford Observatory, and JPL for hospitality. This work was supported at the University of Washington by NSF.

[1] Hogan, C. J. 2000, Phys. Rev. Lett. 85, 2044

[2] Applegate, J. and Hogan, C. J. 1985, Phys. Rev. D 31, 3037

[3] R. A. Malaney and G. J. Mathews, Phys. Rep. 229, 1445, 1993

[4] Kainulainen, K., Kurki-Suonio, H., and Sihvola, E. Phys.Rev. D59, 083505 (1999)

[5] Lange, A. E. et al. 2000, ApJ submitted, astro-ph/0005004

[6] Heckler, A. and Hogan, C. J., Phys. Rev. D 474256 (1993)

[7] Arkani-Hamed, N., Dimopoulos, S., and Dvali, G. Phys. Lett. B429, 263 (1998)

[8] Antoniadas, I., Arkani-Hamed, N., Dimopoulos, S., and Dvali, G., Phys. Lett. B436, 257 (1998)

[9] Arkani-Hamed, N., Dimopoulos, S., and Dvali, G. Phys. Rev. D59, 086004 (1999)

[10] Randall, L. and Sundrum, R., Phys.Rev.Lett. 83, 3370 (1999)

[11] Randall, L., and Sundrum, R., Phys. Rev. Lett. 83, 460 (1999)

[12] Lykken, J. and Randall, L., J. High En. Phys. 0006, 014 (2000)

[13] Hoyle, C. D., Heckel, B., Adelberger, E., Schmidt, U., Gundlach, J., Swenson, E., \& Kapner, D., in preparation (2000)

[14] Goldberger, W. D. and Wise, M., "Phenomenology of a Stabilized Modulus," hep-ph/9911457

[15] Kaplan, D. B. and Wise, M., "Couplings of a Light Dilaton and Violations of the Equivalence Principle", hep-ph/0008116

[16] Kolb, E. W. and Turner, M. S., The Early Universe, (Addison-Wesley,1990)

[17] Hogan, C. J., "Cosmological Gravitational Wave Backgrounds," in Second International LISA Symposium on the Detection and Observation of Gravitational Waves in Space, AIP conference proceedings 456, ed. W. M. Folkner, pp. 79-86 (1999), astro-ph/9809364

[18] Maggiore, M., "Gravitational Wave Experiments and Early Universe Cosmology", Phys. Rep. in press (2000), gr-qc/9909001

[19] Maggiore, M., "Stochastic Backgrounds of Gravitational Waves", gr-qc/0008027

[20] LISA Study Team, LISA Pre-Phase A Report (1998); this document and others are available at http://lisa.jpl.nasa.gov/documents/

[21] Armstrong, J. W., Estabrook, F. B., and Tinto, M., Astrophys. J. 527, 814 (1999)

[22] Allen, B., in Les Houches School on Astrophysical Sources of Gravitational Waves, eds. Jean-Alain Marck and Jean-Pierre Lasota, (Cambridge University Press, 1996); gr-qc/9604033

[23] K. Thorne, private communication

[24] Zel'dovich, Ya. B., Kobzarev, I. Yu. and Okun, L. B., Zh. Eksp. Teor. Fiz. 67, 3 (1974)[Sov. Phys. JETP 40, 1 (1975)].

[25] Kibble, T. W. B., J. Phys. A 9, 1387 (1976)

[26] Vilenkin, A., Phys. Rev. Lett. 48, 59 (1982)

[27] Krauss, L. M., Phys. Lett. B 284, 229 (1992) 
[28] Hogan, C. J., Phys. Rev. Lett. 74,3105 (1995)

[29] Witten, E., Phys. Rev. D 30, 272 (1984)

[30] Hogan, C. J., Mon. Not. Roy. Astron. Soc. 218, 629 (1986)

[31] Kamionkowski, M., Kosowsky, A., and Turner, M. S., Phys.Rev. D 49, 2837 (1994)

[32] Gleiser,M. and Roberts, R., Phys.Rev.Lett. 81, 5497 (1998)

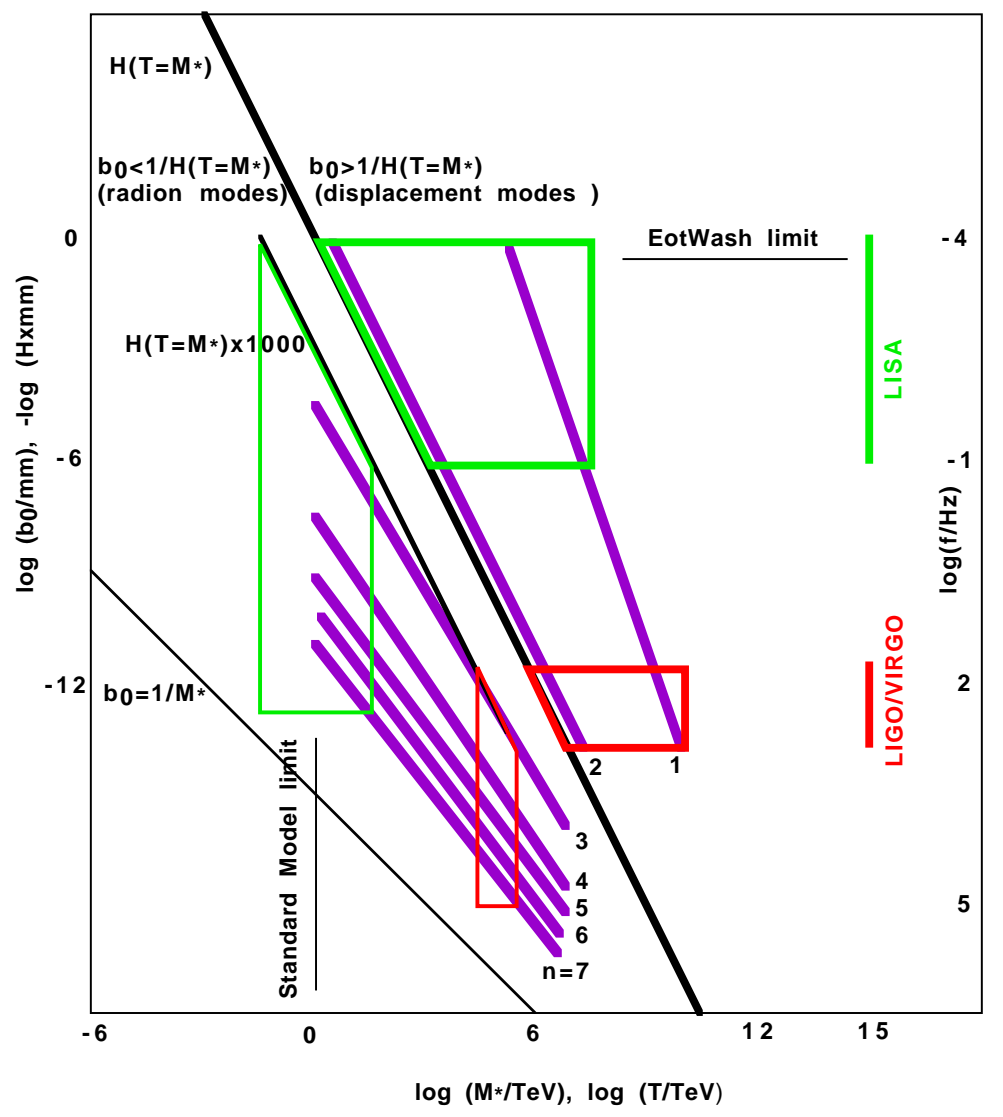

FIG. 1. Summary of the new parameter space of extra dimensions that will be probed by gravitational-wave interferometers. In the context of models where $n$ extra dimensions have the same size $b_{0}$ and the rest lie at the unification scale $M_{*}^{-1}$, a choice of $n$ defines the relation between $M_{*}$ and $b_{0}$; these relations are shown labeled by $n$. Classical, $3+1-\mathrm{D}$ relativistic cosmology (the Friedmann equation) defines the relation $H\left(T=M_{*}\right)$ as shown. Theories with $b_{0} \geq 1 / H\left(T=M_{*}\right)$ condense their classical 3-brane before the Hubble length is as large as $b_{0}$, and the random location of the brane in the extra dimensions excites Nambu-Goldstone displacement modes; the rms strain peaks near the frequency $f_{H 0}\left(H=b_{0}^{-1}\right)$ and is damped at lower frequencies. Theories with $b_{0} \leq 1 / H\left(T=M_{*}\right)$ on the other hand are already $3+1-\mathrm{D}$ on the Hubble scale when they pass the unification temperature; the final stabilization of the extra dimensions then appears as a phase transition in a 3+1-D cosmology, with an order parameter represented by the radion. The strain in this case peaks at a frequency determined by the nucleation scale, typically at least 100 times higher than $f_{H 0}\left(T=M_{*}\right)$; the relation $H\left(T=M_{*}\right) \times 1000\left(\right.$ or $\left.f=1000 \times f_{H 0}\left(T=M_{*}\right)\right)$ shows the peak frequency for an illustrative radion background. Boxes indicate the corresponding regions of these parameters which may give rise to detectable mesoscopic gravitational radiation backgrounds in the LISA and LIGO bands. Heavy-line boxes show the displacement mode parameters, lighter-line boxes show the radion mode parameters. These regions extend well beyond those already constrained by gravitational experiments, direct particle production, or other astrophysical constraints. Theories which "solve the hierarchy problem" have $M_{*}$ close to the Standard Model limit, and all of the viable ones $(2 \leq n \leq 7)$ could possibly produce an observable background of one type or the other in the LISA band. 